\title{
Ear Cavity Biometric Recognition
}

\author{
Ms. Bindhu K. Rajan, Mr. Rijo P C \\ Electronics and Communication Engineering Jyothi Engineering College Cheruthuruthy, Thrissur, \\ Kerala, India \\ Melon Jobi, Sajin Thomas K, Smrithi K H, Veena C V, V S Anjali \\ Electronics and Communication Engineering Jyothi Engineering College Cheruthuruthy, Thrissur, \\ Kerala, India
}

\begin{abstract}
With the increasing concerns on security breach and transaction fraud, highly reliable and convenient personal verification and identification technologies are more and more requisite in our social activities and national services. Biometrics, in which the distinctive physiological and behavioural characteristics are used to recognize the identity of an individual, are gaining ever-growing popularity in an extensive array of governmental, military, forensic, and commercial security applications. Here, the resonation of sound is determined by the shape of human ear cavities to distinguish individuals. This system works measuring the acoustic characteristics determined by the shape of the ear. This is unique to individuals. It uses an earphone with a built-in microphone. This collects earphone-generated sounds as they resonate with ear cavities. It is then processed and an average of multiple signals is obtained. Average frequency domain spectrum for a single person is stored in the database. To compare similarity between signals we use the cross correlation. In Matrix Laboratory (MATLAB) the similarities between the signals are compared. Then for the identification the database is correlated with the currently recorded sound for matching.
\end{abstract}

Index Terms: Ear cavity, biometric, matching, MATLAB

\section{Introduction}

In everyday and social activities reliable personal recognition techniques play a critical role. Authorized users should be allowed for entrance with high accuracy while unauthorized users should be denied, in access control. People not only should identify whether the identity of a person is whom he/she claimed to be, but also should avoid the occurrence that one person claims to be another person to receive the welfare benefit twice, in welfare benefit disbursement. Biometrics in which the distinctive physiological and behavioral characteristics are used to recognize the identity of an individual. These are gaining ever-growing popularity in an extensive array of governmental, military, forensic, and commercial security applications.

From when fingerprint has been used for forensic, the beginning of biometrics can be traced back to centuries ago. Automated biometrics, however, has only 40 years of history. The FBI began to put more effort in developing automated fingerprint acquisition and identification systems, in the early 1960's. With the advances in hardware, sensor, pattern recognition, signal and image processing technologies, a number of biometric technologies, such as face, iris, retina, voice, signature, hand geometry, keystroke, ear, and palm print recognition, have been developed, and novel biometrics such as dental, odour,and skin reflectance, have also been investigated to overcome some of the limitations of current biometric recognition technologies. The four major modules in biometric system include: data acquisition, feature extraction, matching, and system database. Here, feature extraction and matching are two of the most challenging problems of biometric recognition research. And this has attracted researchers from different backgrounds like biometrics, computer vision, pattern recognition, signal processing, and neutral networks. The ear cavity biometric recognition instantaneously measures (within approximately one second) acoustic characteristics determined by the shape of the ear, which is unique to each person, using an earphone with a built-in microphone to collect earphone-generated sounds as they resonate with ear cavities. This unique method of extracting features is useful for distinguishing individuals based on acoustic characteristics and enables rapid and highly accurate recognition with accuracy greater than 99 percentage .Biometrics is currently being used in areas such as computer desktops, networks, banking, immigration, law enforcement, telecommunication networks and monitoring the time and attendance of staff.

\section{Literature Survey}

This section contains some earlier works for authentication using ear biometrics and audio pattern matching. Different algorithms are used in papers discussed below. These algorithms have lack of accuracy and efficiency and they take more time to process, and thus less portable. Paper [1] shows, although acoustics of the external ear has been studied extensively for auralization and hearing aids, the acoustic behavior with insert headphones is not as well known. The effects of outer ear physical dimensions, particularly to sound pressure in 
the eardrum are what our research focused on. Eardrum's damping of resonances was studied, but the main parameter were the length of the canal. Two ear canal simulators and one dummy head were constructed. Measurements were also performed from human ear canals. Both with unblocked ear canals and when the canal entrance was blocked with an insert earphone, the study was carried out. Special inserts earphones with in-ear microphones were constructed for this purpose. Physics-based computational models were finally used to validate the approach.

It is well known that the outer ear contributes to the spectral shaping of sounds we hear of everyday life. People have different ears and different ear canals, hence the sound pressure responses to people's eardrums are not similarly distributed. In part therefore, people perceive sounds differently. In normal listening situations the whole outer ear contributes to the spectral shaping of sounds before they reach the eardrum. The unblocked ear canal acts like a quarter-wave resonator and hence amplifies the resonance frequencies. The locations of these resonance frequencies of the frequency domain depend mainly on the effective length of the ear canal. The shape and size of the pinna, and the curvature of the ear canal also has an effect on the pressure frequency response to the eardrum. Insert type earphones is increasingly popular when listening to music and together with mobile phones, yet their behavior has not been studied thoroughly. The sound transmission path of the insert earphone to the eardrum is different from listening to loudspeakers or acoustically open headphones. The sound wave travels through the ear canal only, an ear canal that is suggestive of a half-wave resonator. The half-wave resonance frequencies are pronounced at the eardrum, and the locations of these frequencies depend once again on the length of the ear canal. In addition, the overall structure of the ear canal has an effect on the shape of the frequency response to the eardrum. Furthermore, the pressure chamber effects and the occlusion effects (emphasis of own voice) are important factors regarding insert earphones. In order to measure the effects of the differences in physical parameters of the outer ear a variety of different ear canal simulators, different kinds of artificial pinna, and a dummy head was constructed. The main focus of this study was on the effect of the length of the ear canal and the effect of the eardrum impedance The acoustic behavior of the outer ear and the ear canal was studied through extensive measurements with ear canal simulators, the dummy head, and human test subjects. The goal was to learn how the outer ear behaves with and without insert earphones. For measuring the frequency responses of a blocked ear canal, a special earphone was constructed. A Knowles FG23329 miniature microphone was fitted in front of the transducer port of a Philips SHN2500 earphone as depicted in Figure 4. When the acoustic behavior of a blocked ear canal or ear canal simulator was studied, this Earphone With Fitted In-Ear Microphone (EFIM) was placed at the canal entrance. Impulse response measurements were made with $\log$ sine sweep technique. Paper [2] represents a method of recognizing the sounds in the environmental. The scene surrounding an audio sensor can be understood. For audio recognition, a variety of features are proposed. It includes the popular Mel-frequency cepstral coefficients (MFCCs). MFCCs describes the audio spectral shape. Environmental sounds includes chirpings of insects and sounds of rain. They are noise-like with a broad flat spectrum. It include strong temporal domain signatures. Only few temporaldomain features have been developed. This characterize the diverse audio signals. We also perform an empirical feature analysis for audio environment characterization. It is proposed to use the matching pursuit (MP) algorithm. It results in an effective time-frequency features. The MP-based method utilizes a dictionary of atoms for feature selection. This gives a flexible, intuitive and physically interpretable set of features. The MPbased feature is adopted to supplement the MFCC features. This yields higher recognition accuracy for environmental sounds. Extensive experiments are conducted. This demonstrates the effectiveness of these joint features. This is for unstructured environmental sound classification. This includes listening tests to study human recognition capabilities.

\section{Proposed Methodology}

The ear cavity biometric recognition method is used for authentication purpose. It is mainly based on the resonation of sound determined by the shape of the human ear cavity. A few hundred milliseconds of acoustic signals ranging from $20 \mathrm{~Hz}$ to $20 \mathrm{KHz}$ are produced by the ear phone. The signals are resonated within the ear cavity and is received through the microphone. The average of multiple signals are selected after processing. The acoustic characteristics determined by the shape of the ear. The recorded reflected sound is processed in MATLAB software. The frequency domain spectrum is extracted. The frequency domain spectrum of the produced sound is plotted.

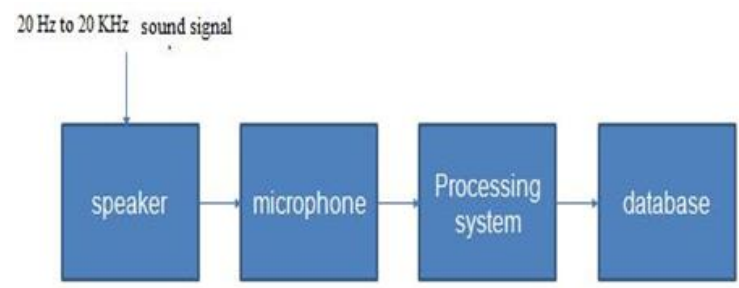

Fig. 1. Block Diagram 
Using the database, the average frequency domain spectrum for an individual is stored. The database is then correlated with the recorded sound for further matching and authentication. The similarity between the signals is observed using cross correlation.

\section{Hardware Implementataion}

Hardware components used are earphone and microphone. An earphone is used to transmit the signals to the ear cavity. The resonated sound is then collected using the embedded microphone. The earphone and the MIC are incorporated together as a device. The device is then made wearable for the user and is made comfortable for authentication purpose. Database of recorded sounds are created which is used for authentication using MATLAB and VisualBaiscs.

\section{Speaker}

Device emitting audible sound ranging from $20 \mathrm{~Hz}$ to

20KHz.

\section{Microphone}

Microphone is a transducer. It converts sound into an electrical signal. Microphones are used in many applications. It includes telephones, hearing aids, public address systems for concert halls and public events, motion picture production, live and recorded audio engineering, etc. Several different types of microphones are used. Different methods are used to convert the air pressure variations of a sound wave to an electrical signal. Dynamic microphones are most commonly used. Vibrating diaphragm is used as a capacitor plate. The piezoelectric microphone uses a crystal of piezoelectric material. Microphones typically need to be connected to a preamplifier. And then the signal is recorded or reproduced.

\section{Processing System}

A combination of machines, people, and processes represents the data processing system. A set of outputs are produced as a result inputting a set of inputs. The inputs and outputs are interpreted as data, facts, information. It depend on the interpreter's relation to the system. MATLAB (matrix laboratory) is a multiparadigm numerical computing environment. MATLAB is a fourth-generation programming language. It is a proprietary programming language developed by Math Works. Functions of MATLAB includes matrix manipulations, plotting of functions and data, implementation of algorithms, creation of user interfaces, and interfacing with programs written in other languages, including C, C++, Java, Fortran and Python. MATLAB is intended primarily for numerical computing. An optional toolbox uses the Mu PAD symbolic engine which allows access to symbolic computing abilities.

Simulink is an additional package. It adds graphical multi-domain simulation and model-based design. It is mainly used in dynamic and embedded systems. MATLAB scripting language has a built-in MATLAB application. The Command Window is used as an interactive mathematical shell. Also, executes text files containing MATLAB code.

(a) Creating Database

\section{Software Implementation}

Database was created by recording the sound ranging from $20 \mathrm{~Hz}-20 \mathrm{KHz}$. Saved the recorded sound as a MAT file.

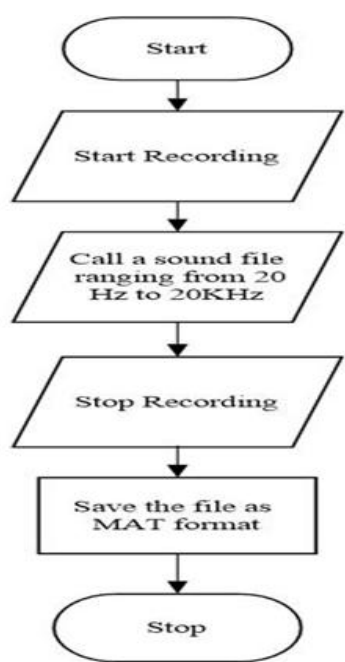

Fig 2: Flowchart 
(b) Authentication

Started recording. Then the sound was played. Stopped recording. The recorded data was moved into a variable. Moved all stored sound data into different variables. Plotted each of the sound signals using the above data. Correlated the presently recorded signal with the signal stored earlier and stored the correlation variable into another variable. The above steps were repeated for the remaining signals. Maximum value from the array was taken which was formed by correlating the recorded signal and and stored the result in a variable.

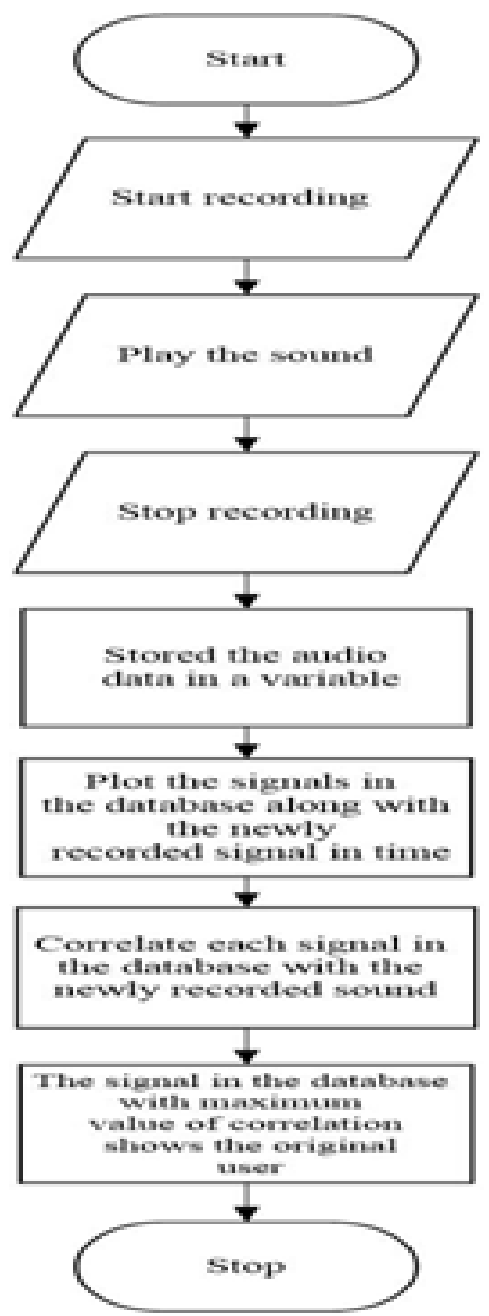

Fig 3: Flowchart

Flow chart for the proposed system is explained in the Fig. 3. It shows the way of comparing two signals for authentication.

\section{Conclusion}

A biometric technology capable of determining the identity of a person is obtained. Individuals are recognized based on the resonation of sound determined by the shape of human cavities. The new technology instantaneously measures acoustic characteristics determined by the shape of the ear which is unique for individuals. It enables a stable and rapid means for authentication. Highly accurate recognition is obtained. This method of recognition gives all the comfort to people.

\section{References}

[1]. Hiipakka, Marko, Miikka Tikander, and Matti Karjalainen. "Modeling the external ear acoustics for insert headphone usage." Journal of the Audio Engineering Society 58.4 (2010): 269-281

[2]. Chu, Selina, Shrikanth Narayanan, and C-C. Jay Kuo. "Environmental sound recognition with time-frequency audio features." IEEE Transactions on Audio, Speech, and Language Processing 17.6 (2009): 1142-1158.

[3]. Wang, Yu-Duo, and Li Bo. "Integrations between Visual Basic and MATLAB." Computational Intelligence and Communication Networks (CICN), 2013 5th International Conference on. IEEE, 2013. 